\title{
Global post-marketing safety surveillance of Tumor Treating Fields (TTFields) in patients with high-grade glioma in clinical practice
}

\author{
Wenyin Shi ${ }^{1}$ (D) Deborah T. Blumenthal ${ }^{2} \cdot$ Nancy Ann Oberheim Bush ${ }^{3} \cdot$ Sied Kebir ${ }^{4,5} \cdot$ Rimas V. Lukas $^{6,7}$. \\ Yoshihiro Muragaki $^{8,9} \cdot$ Jay-Jiguang Zhu ${ }^{10} \cdot$ Martin Glas $^{4,5}$
}

Received: 8 April 2020 / Accepted: 21 May 2020

(c) The Author(s) 2020, corrected publication 2020

\begin{abstract}
Introduction Tumor Treating Fields (TTFields; antimitotic treatment) delivers low-intensity, intermediate-frequency, alternating electric fields through skin-applied transducer arrays. TTFields $(200 \mathrm{kHz})$ was FDA-approved in glioblastoma (GBM), based on the phase 3 EF-11 (recurrent GBM, rGBM) and EF-14 (newly diagnosed GBM, ndGBM) trials. The most common TTFields-related adverse event (AE) in both trials was array-associated skin irritation. We now report on TTFields-related AEs in the real-world, clinical practice setting.

Methods Unsolicited, post-marketing surveillance data from TTFields-treated patients (October 2011-February 2019) were retrospectively analyzed using MedDRA v21.1 preferred terms, stratified by region (US, EMEA [Europe, Middle East, Africa], Japan), diagnosis (ndGBM, rGBM, anaplastic astrocytoma/oligodendroglioma, other brain tumors), and age $(<18$ [pediatric], 18-64 [adults], $\geq 65$ [elderly]; years of age).

Results Of 11,029 patients, 53\% were diagnosed with ndGBM and 39\% were diagnosed with rGBM at any line of disease recurrence. Most were adults (73\%), 26\% were elderly, and the male-to-female ratio was 2:1 (close to published ratios of typical GBM populations). The most commonly reported TTFields-related AE was array-associated skin reaction, occurring in patients with ndGBM (38\%), rGBM (29\%), anaplastic astrocytoma/oligodendroglioma (38\%), and other brain tumors (31\%); as well as $37 \%$ of pediatric, $34 \%$ of adult, and $36 \%$ of elderly patients. Most skin AEs were mild/moderate and manageable. Other TTFields-related AEs in patients with ndGBM/rGBM included under-array heat sensation (warmth; 11\%, $10 \%$, respectively) and electric sensation (tingling; $11 \%, 9 \%$, respectively), and headache ( $7 \%, 6 \%$, respectively).

Conclusions This TTFields safety surveillance analysis in $>11,000$ patients revealed no new safety concerns, with a favorable safety profile comparable with published TTFields/GBM trials. The safety profile remained consistent among subgroups, suggesting feasibility in multiple populations, including elderly patients.
\end{abstract}

Keywords TTFields $\cdot$ Glioblastoma $\cdot$ Real-world $\cdot$ Safety surveillance $\cdot$ Tolerability $\cdot$ Skin adverse events

\section{Introduction}

The most common primary brain/central nervous system (CNS) cancers in adults are malignant gliomas, including glioblastoma (GBM), anaplastic astrocytoma, and anaplastic oligodendroglioma [1]. GBM is an aggressive glioma that accounts for $15 \%$ of all primary brain/CNS tumors, $48 \%$ of primary malignant CNS tumors, and $57 \%$ of all gliomas in the United States (US) [2]. Although GBM is a rare tumor type with an estimated global GBM incidence of $<10$ per

Wenyin Shi

Wenyin.Shi@jefferson.edu

Extended author information available on the last page of the article
100,000 , it has high mortality and an extremely poor prognosis [3, 4]. Moreover, GBM incidence is generally higher in men, with a male-to-female ratio ranging from 1.0 to 1.9 $[2,5]$. In 2020, 13,140 new cases of GBM are projected in the US, including 6950 cases among people $\geq 65$ years of age [2].

Prior to Tumor Treating Fields (TTFields), the first-line, standard of care (SOC) treatment for patients with newly diagnosed GBM (ndGBM) consisted of maximal safe resection followed by adjuvant radiotherapy (RT) plus concomitant and maintenance temozolomide (TMZ) chemotherapy [6-8]. Median overall survival (OS) for GBM ranges from 6.0 to 19.6 months, despite treatment advances in neurosurgery, RT, and chemotherapy [9]. The 1-year survival rate 
is $41 \%$, ranging from $72 \%$ in patients who are $20-44$ years of age to $31 \%$ in patients who are $65-74$ years of age [2]. Based on positive phase 3 results, the new SOC treatment algorithm for ndGBM includes the addition of TTFields to maintenance TMZ $[8,10,11]$. In general, TTFields are a novel, noninvasive, antineoplastic therapy involving localized delivery of low-intensity $(1-3 \mathrm{~V} / \mathrm{cm})$, intermediatefrequency $(100-500 \mathrm{kHz})$, alternating electric fields that affect rapidly dividing cancer cells [12-15]. The fields are delivered continuously to the locoregional tumor bed using 2 pairs of skin-affixed transducer arrays.

Based on the phase 3 EF-11 and EF-14 clinical trial data, the US Food and Drug Administration (FDA) approved TTFields (200 kHz optimal frequency for GBM) for adults ( $\geq 22$ years of age) as monotherapy for recurrent GBM (rGBM; 2011) and in combination with adjuvant postchemoradiation TMZ for ndGBM (2015). In addition to US approval, global ndGBM and rGBM approvals for TTFields in adults ( $\geq 18$ years of age) include Conformitè Europëenne (CE) Mark IIB in various Europe, Middle East, and Africa (EMEA) regions, the Pharmaceuticals and Medical Devices Agency (PMDA) Class III in Japan, and most recently (2020) by the National Medical Products Administration (NMPA) in China.

Notably, TTFields is classified as a category 1 recommendation in the National Comprehensive Cancer Network ${ }^{\circledR}$ guidelines for ndGBM [10] and is recognized as a treatment advancement in clinical cancer care by the American Society of Clinical Oncology [16]. Although the scope of guidance by the American Society of Radiation Oncology (2016) is on evidence-based radiotherapy or concomitant treatments to radiotherapy, TTFields was mentioned albeit limited published trial data at time of clinical evidence assessments for guideline inclusions (i.e., implemention of a priori exclusion of studies available only as abstracts). A planned, global clinical trial (TRIDENT) will assess the role of TTFields as an upfront treatment in combination with radiochemotherapy. European guidelines which incorporate TTFields include Spain's Spanish Society of Medical Oncology (2017), England's National Institute for Health and Care Excellence (2018), as well as the Swedish national guidelines for tumors in the brain and spinal cord (2020) that specifically endorses the addition of TTFields for GBM. European Society of Medical Oncology clinical practice guideline (2014) mentions TTFields, although the guideline is currently outdated, as it predates current clinical evidence available for TTFields. Moreover, the Society of Neuro-oncology and European Society of Neuro-Oncology joint consensus (2020) has described TTFields as a treatment that improves survival in ndGBM. Other global guidelines include China's Glioma Treatment Guidelines (2018), which recommends TTFields for patients with GBM based on Level 1 evidence.
TTFields demonstrated efficacy in patients with ndGBM in the phase 3 EF-14 clinical trial [11]. Median progression-free survival was significantly improved to 6.7 months with TTFields plus TMZ versus 4.0 months with TMZ alone (hazard ratio [HR], $0.63 ; 95 \%$ confidence interval $[\mathrm{CI}], 0.52-0.76 ; \mathrm{p}<0.001)$. Median OS was significantly improved to 20.9 months versus 16.0 months from randomization (or from 24.5 months versus 19.8 months from diagnosis), respectively (HR, 0.63 ; 95\% CI, 0.53-0.76; $p<0.001)$ [11]. The 5-year survival rate was $13 \%$ versus $5 \%$, respectively $(p=0.004)$, a 2.6-fold increase [11]. In post hoc analyses of the EF-14 clinical trial, TTFields plus TMZ in all patient subgroups was associated with increased PFS and OS (Cox proportional hazards; $p<0.05$ for the treatment effect within each subgroup), regardless of age, sex, Karnofsky performance score (KPS), O6-methylguanine-DNA methyltransferase promoter methylation status, geographic region, or extent of resection [11].

GBM almost universally recurs. Prior to TTFields, phase 2 and 3, rGBM clinical trials reported median OS after recurrence of only 6.0-9.8 months [17-23]. However, a post-hoc EF-14 analysis found that TTFields plus TMZ after first recurrence could prolong median OS versus TMZ alone for patients that were treated with TTFields beyond first progression (11.8 vs 9.2 months, respectively; HR, 0.70; 95\% CI, 0.48-1.00; $\mathrm{p}=0.049$ ) [24].

TTFields was well-tolerated by patients in phase 3 trials; the most common treatment-related adverse event (AE) was localized skin AEs beneath the arrays [11, 21]. In the EF-14 trial, the majority of skin AEs (52\%) were grade 1 or 2 (mild-to-moderate) and only $2 \%$ were grade 3 (severe) [11]. Systemic AE rates were similar with TTFields plus TMZ versus TMZ alone (48\% vs $44 \%$, respectively) and were consistent with previously reported TMZ clinical trials [7, 11, 25-27].

The most common AE in the Patient Registry Dataset (PRiDe), a post-marketing registry of 457 patients with rGBM who received TTFields in the US (October 2011-November 2013), was also array-associated mildto-moderate skin irritation [28]. No new TTFields-related safety signals or systemic AEs were noted [28]. Overall, TTFields-related skin AEs based on registry were easily managed and treated with topical corticosteroids or antibiotics $[21,28]$ and typically did not require treatment interruption $[21,28]$.

This retrospective, unsolicited, global, post-market surveillance study aims to expand the real-world safety evidence for TTFields by analyzing AE profiles from a cohort of $>11,000$ patients who received TTFields in the real-world, clinical practice settings within the US, EMEA, and Japan. 


\section{Methods}

Safety data were retrospectively collected from routine, post-marketing surveillance of patients with brain tumors treated with TTFields (October 2011-February 2019). AE data were not actively solicited, but collated from published literature screening and patient, caregiver, and/or prescriber reports during routine interactions with the device manufacturer (Novocure ${ }^{\circledR}$; e.g., Device Support Specialist visits, prescriber interactions, and patient emails to nCompass ${ }^{\mathrm{TM}}$ support team). Subsequently, AEs were assessed by the Medical Safety Department as mandated by health authorities. AE reporting was based on the Medical Dictionary for Regulatory Activities version 21.1 (MedDRA v21.1) body system organ classes and preferred terms. Data were collected from patients treated with TTFields in the US, EMEA, and Japan. A subset of this data was previously published in the PRiDe registry. Safety data was provided by device manufacturer for current safety analyses. Since data were retrospectively gathered, AE severity could only be classified as nonserious or serious. An AE was considered serious if it led to $\geq 1$ of the following: (1) death; (2) life-threatening illness/ injury; (3) permanent body structure/function impairment; (4) in-patient hospitalization or prolongation of existing hospitalization; (5) medical/surgical intervention to prevent life-threatening illness, injury, or permanent body structure/function impairment; (6) fetal distress/ death, congenital abnormality, or birth defect.

\section{Patients and treatment}

Patients were 3-89 years of age and included those with brain tumors treated with TTFields between October 2011 and February 2019 in the US, EMEA, and Japan. Information on TTFields in combination with other cancer/ concomitant treatments was not included, as data were collated from routine, retrospective, post-marketing surveillance reports, and combination data were not available for all patients. Hence, findings derived from these safety data cannot be deemed conclusive due to their observational nature. However, due to the large sample size, analyses were conducted to screen for new safety signals or trends relating to TTFields in the real-world clinical setting. Therefore, some patients may have received TTFields in combination with chemotherapy, immunotherapy, anti-vascular endothelial growth factor agents, and/or other concomitant treatments. It is important to note that safety effects of other treatments could not be adjusted for and should be considered when evaluating these data.

\section{Analyses}

Data from the overall patient cohort were stratified by: (1) diagnosis, including ndGBM, rGBM, as well as off-label use for grade 3 glioma (anaplastic astrocytoma and anaplastic oligodendroglioma) and other brain tumors (including brain metastases); (2) region (US, EMEA, and Japan); and (3) age group (3 to 17 years of age, pediatric [off-label use]; 18 to 64 years of age [in the US, off-label for ages 18-21], adult; and 65 to 89 years of age, elderly). Novocure's Medical Safety department assessed AE relatedness to treatment; incidence was based on the number of unique patients reporting an AE. Moreover, due to the retrospective study design, additional types of patient information could not be analyzed. This was largely due to the non-prespecified nature of innate study design, in which patient AE reports were unsolicited. Also, routine, global post-marketing surveillance activities have no uniform mandate, as they often lack additionally collected patient information and separate documentation.

\section{Results}

\section{Baseline characteristics}

Safety reports were received during post-marketing surveillance of 11,029 patients treated with TTFields. The majority of TTFields-treated patients were diagnosed with ndGBM (53\%) or rGBM (39\%) (Table 1). Median age at TTFields initiation was 57 years of age (range: 3-89). Approximately three-quarters $(73.4 \%)$ of patients were adults $(\geq 18$ to $<65$ years of age), and $26.2 \%$ were elderly ( $\geq 65$ years of age; Table 1). Although TTFields are not currently approved for pediatric use, $52(0.5 \%)$ pediatric patients were identified in the total cohort. Age stratification of total cohort showed that the most common diagnosis among pediatric patients was rGBM $(42.3 \%)$, while the majority of adult $(50.2 \%)$ and elderly $(62.5 \%)$ patients were diagnosed with ndGBM (Table 1). Few patients $(\sim 7 \%)$ were treated off-label with TTFields for anaplastic astrocytoma and anaplastic oligodendroglioma or other brain tumors (including brain metastases from different cancer types). Regionally, $78.2 \%$ of patients were treated in the US, $20.4 \%$ in EMEA regions, and $1.4 \%$ in Japan. In all three regions, more patients with ndGBM than rGBM received treatment with TTFields (Fig. 1). The very high rate of ndGBM diagnoses in Japan (97.4\%) is likely a result of Japan's national government health insurance only covering reimbursement for ndGBM treatment, despite approval of TTFields for ndGBM and rGBM treatment by the Ministry of Health, Labour and Welfare in Japan. Overall, the 1.97:1 ( 2:1) male-to-female patient ratio $(66.3 \%$ [ $\mathrm{n}=7313$ ] to $33.7 \%[\mathrm{n}=3716]$, respectively), although on 
Table 1 Baseline characteristics of the TTFields-treated population $(\mathrm{N}=11,029)$

\begin{tabular}{llllll}
\hline Characteristic & Total & ndGBM & rGBM & AA/AO & Other $^{\text {a }}$ \\
\hline $\begin{array}{llll}\text { N/n (\%) } \\
\text { Age (years), n (\%) }\end{array}$ & $\mathrm{N}=11,029(100.0)$ & $\mathrm{n}=5887(53.4)$ & $\mathrm{n}=4345(39.4)$ & $\mathrm{n}=682(6.2)$ & $\mathrm{n}=115(1.0)$ \\
$\quad<18$ & $52(0.5)$ & $19(0.3)$ & $22(0.5)$ & $8(1.2)$ & $3(2.6)$ \\
18 to 64 & $8090(73.4)$ & $4063(69.0)$ & $3337(76.8)$ & $596(87.4)$ & $94(81.7)$ \\
$\geq 65$ & $2887(26.2)$ & $1805(30.7)$ & $986(22.7)$ & $78(11.4)$ & $18(15.7)$ \\
Gender, n (\%) & & & & & \\
Male & $7313(66.3)$ & $3849(65.4)$ & $2921(67.2)$ & $470(68.9)$ & $73(63.5)$ \\
Female & $3716(33.7)$ & $2038(34.6)$ & $1424(32.8)$ & $212(31.1)$ & $42(36.5)$ \\
Region, n (\%) & & & & & \\
United States & $8628(78.2)$ & $4402(74.8)$ & $3583(82.5)$ & $540(79.2)$ & $103(89.6)$ \\
EMEA & $2248(20.4)$ & $1336(22.7)$ & $758(17.4)$ & $142(20.8)$ & $12(10.4)$ \\
Japan & $153(1.4)$ & $149(2.5)$ & $4(0.1)$ & 0 & 0 \\
\hline
\end{tabular}

Note: all proportions do not equal $100 \%$, due to rounding to the tenths decimal

$A A$ anaplastic astrocytoma, $A O$ anaplastic oligodendroglioma, EMEA Europe, the Middle East, and Africa, ndGBM newly diagnosed glioblastoma, $r G B M$ recurrent glioblastoma, TTFields Tumor Treating Fields

${ }^{a}$ Other brain tumors including brain metastases from different cancer types

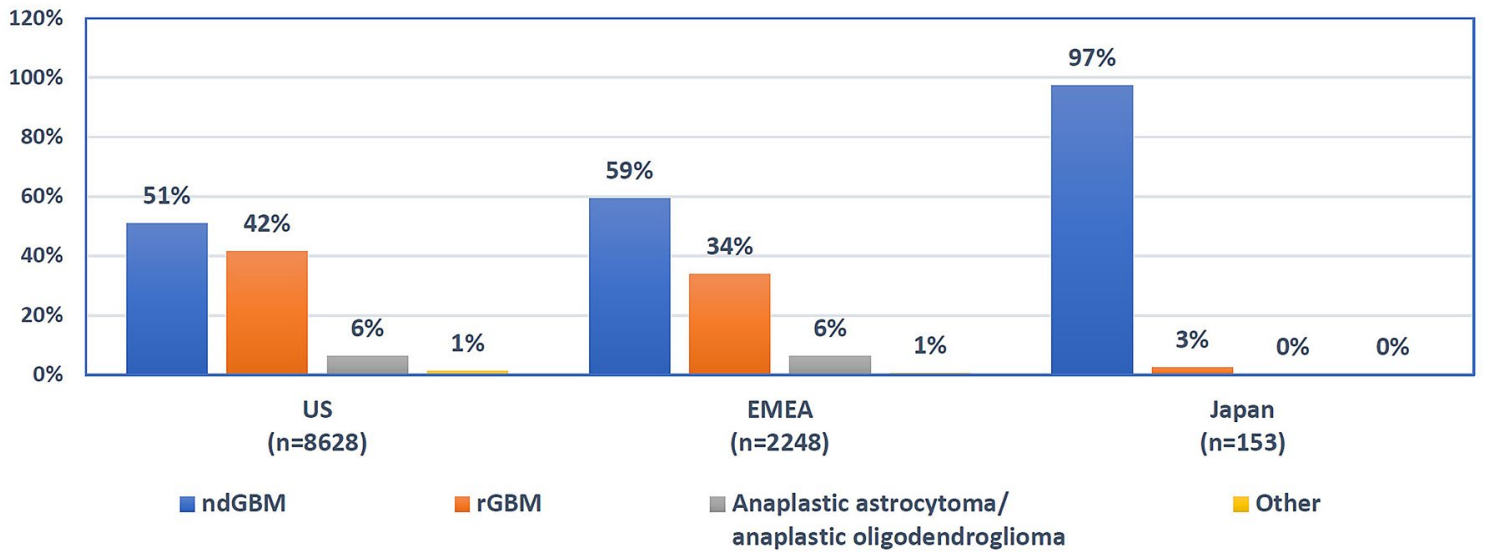

Fig. 1 Proportion of patients who received TTFields by diagnosis and region. EMEA Europe, the Middle East, and Africa, $n d G B M$ newly diagnosed glioblastoma, rGBM recurrent glioblastoma, TTFields

the higher-end of range, was closely representative of published ratios of a typical GBM population $[2,5]$.

\section{Safety}

Overall, 63\% of patients treated with TTFields reported $\geq 1$ AE (Table 2). Stratification by diagnosis showed similar proportions of patients with ndGBM (65\%), rGBM (60\%), anaplastic astrocytoma and anaplastic oligodendroglioma $(65 \%)$, and other brain tumors (61\%) reporting $\geq 1 \mathrm{AE}$. When stratified by age, $58 \%$ of pediatric, $63 \%$ of adult, and $66 \%$ of elderly patients reported $\geq 1$ AE. In general, AEs with a reported incidence of $\geq 5 \%$ in the total cohort were comparable across diagnostic and age subgroups. Skin disorders (36\%), general disorders/application site conditions
Tumor Treating Fields, US United States. ${ }^{a}$ Other brain tumors, including brain metastases from different cancer types

(31\%), and nervous system disorders (27\%) were the most commonly reported AEs among all patients, occurring at analogous rates across diagnostic and age subgroups. General physical health deterioration rates were similarly low across diagnostic and age subgroups ( $0 \%-4 \%$; data not shown).

Skin reactions beneath the transducer arrays were the most commonly reported TTFields-related AEs (34\%) among the total cohort population (Table 2). The incidence of treatment-related skin reactions was greater in patients with ndGBM versus rGBM ( $38 \%$ vs $29 \%$, respectively), yet rates were consistent for pediatric, adult, and elderly age subgroups $(37 \%, 34 \%$, and $36 \%$, respectively). Other AEs potentially related to TTFields included electric sensation (i.e., under-array tingling; $11 \%$ ), heat sensation (i.e., 
Table 2 Most common AEs in TTFields-treated patients by diagnosis and age, with incidence of $\geq 5 \%$ in the total cohort

\begin{tabular}{|c|c|c|c|c|c|c|c|c|}
\hline \multirow{2}{*}{$\begin{array}{l}\text { MedDRA v21.1 system organ } \\
\text { class/preferred term }\end{array}$} & \multirow[t]{2}{*}{ Total } & \multicolumn{4}{|l|}{ Diagnosis } & \multicolumn{3}{|c|}{ Age (years) } \\
\hline & & ndGBM & rGBM & AA/AO & Other ${ }^{\mathrm{c}}$ & $<18$ & 18 to 64 & $\geq 65$ \\
\hline $\mathbf{N} / \mathbf{n}$ & $\mathrm{N}=11,029$ & $\mathrm{n}=5887$ & $\mathrm{n}=4345$ & $\mathrm{n}=682$ & $\mathrm{n}=115$ & $\mathrm{n}=52$ & $\mathrm{n}=8090$ & $\mathrm{n}=2887$ \\
\hline Patients with $\geq 1$ AE, n (\%) & $6985(63)$ & $3847(65)$ & $2625(60)$ & $443(65)$ & $70(61)$ & $30(58)$ & $5061(63)$ & $1894(66)$ \\
\hline General, n (\%) & $3460(31)$ & $1940(33)$ & $1263(29)$ & $223(33)$ & $34(30)$ & $18(35)$ & $2561(32)$ & $881(31)$ \\
\hline Electric sensation $^{\mathrm{a}}$ & $1172(11)$ & $673(11)$ & $402(9)$ & $77(11)$ & $20(17)$ & $7(13)$ & $948(12)$ & $217(8)$ \\
\hline Fatigue/malaise & $589(5)$ & $353(6)$ & $198(5)$ & $34(5)$ & $4(3)$ & $4(8)$ & $401(5)$ & $184(6)$ \\
\hline Heat sensation ${ }^{\mathrm{b}}$ & $1153(10)$ & $619(11)$ & $439(10)$ & $86(13)$ & $9(8)$ & $9(17)$ & $862(11)$ & $282(10)$ \\
\hline Pain & $670(6)$ & $413(7)$ & $212(5)$ & $41(6)$ & $4(3)$ & $5(10)$ & $475(6)$ & $190(7)$ \\
\hline Injury, n (\%) & $938(9)$ & $477(8)$ & $394(9)$ & $55(8)$ & $12(10)$ & $2(4)$ & $606(7)$ & $330(11)$ \\
\hline Fall & $706(6)$ & $343(6)$ & $311(7)$ & $43(6)$ & $9(8)$ & $2(4)$ & $439(5)$ & $265(9)$ \\
\hline Nervous system, n (\%) & $2924(27)$ & $1571(27)$ & $1160(27)$ & $168(25)$ & $25(22)$ & $14(27)$ & $2145(27)$ & $765(26)$ \\
\hline Headache & $859(8)$ & $467(8)$ & $324(7)$ & $60(9)$ & $8(7)$ & $4(8)$ & $668(8)$ & $187(6)$ \\
\hline Seizure & $1203(11)$ & $631(11)$ & $495(11)$ & $68(10)$ & $9(8)$ & $7(13)$ & $891(11)$ & $305(11)$ \\
\hline Skin, n (\%) & $3985(36)$ & $2345(40)$ & $1334(31)$ & $270(40)$ & $36(31)$ & $19(37)$ & $2888(36)$ & $1078(37)$ \\
\hline Skin reaction & $3805(34)$ & $2251(38)$ & $1258(29)$ & $260(38)$ & $36(31)$ & $19(37)$ & $2748(34)$ & $1038(36)$ \\
\hline
\end{tabular}

$A A$ anaplastic astrocytoma, $A E$ adverse event, $A O$ anaplastic oligodendroglioma, $n d G B M$ newly diagnosed glioblastoma, $r G B M$ recurrent glioblastoma, TTFields Tumor Treating Fields

${ }^{a}$ Commonly described as a tingling sensation beneath the transducer arrays

${ }^{b}$ Local heat beneath the transducer array, commonly described as a warm sensation

${ }^{\mathrm{c}}$ Other brain tumors including brain metastases from different cancer types

under-array warmth; 10\%), and headache (6\%) (Table 2). Electric sensation may be attributable to displaced contact of the transducers arrays on the scalp, which perhaps could result in arching across the gap between the arrays and scalp interface (Table 3). Reports of electric sensations ranged from $8 \%-13 \%$ across age subgroups. Overall, elderly patients, who are $\sim 1 / 3$ of the total sample size that includes pediatric and adult patients, reported the lowest incidences of electric sensations, heat sensations, and headaches across age subgroups (Table 3). Observed lower incidences of electric and heat sensations in elderly are perhaps attributable to less robust hair growth and decreased sweating. Electric sensation was also reported by similar proportions of patients diagnosed with ndGBM and rGBM (11\% and $9 \%$, respectively). Heat sensations were reported in $17 \%$ of pediatric and $10 \%$ of elderly patients. Headaches were

Table 3 Most common TTFields-related AEs with an incidence of $\geq 2 \%$ in total patient cohort, by diagnosis and age

\begin{tabular}{|c|c|c|c|c|c|c|c|c|}
\hline \multirow[t]{2}{*}{ MedDRA v21.1 preferred term } & \multirow[t]{2}{*}{ Total } & \multicolumn{4}{|l|}{ Diagnosis } & \multicolumn{3}{|c|}{ Age (years) } \\
\hline & & ndGBM & rGBM & AA/AO & Other ${ }^{c}$ & $<18$ & 18 to 64 & $\geq 65$ \\
\hline $\mathrm{N} / \mathrm{n}$ & $\mathrm{N}=11,029$ & $\mathrm{n}=5887$ & $\mathrm{n}=4345$ & $\mathrm{n}=682$ & $\mathrm{n}=115$ & $\mathrm{n}=52$ & $\mathrm{n}=8090$ & $\mathrm{n}=2887$ \\
\hline Patients with $\geq 1 \mathrm{AE}, \mathrm{n}(\%)$ & $5260(48)$ & $3022(51)$ & $1835(42)$ & $347(51)$ & $56(49)$ & $27(52)$ & $3842(47)$ & $1391(48)$ \\
\hline Skin reaction & $3805(34)$ & $2251(38)$ & $1258(29)$ & $260(38)$ & $36(31)$ & $19(37)$ & $2748(34)$ & $1038(36)$ \\
\hline Electric sensation $^{\mathrm{a}}$ & $1169(11)$ & $673(11)$ & $399(9)$ & $77(11)$ & $20(17)$ & $7(13)$ & $945(12)$ & $217(8)$ \\
\hline Heat sensation ${ }^{\mathrm{b}}$ & $1153(10)$ & $619(11)$ & 439 (10) & $86(13)$ & $9(8)$ & $9(17)$ & $862(11)$ & $282(10)$ \\
\hline Headache & $716(6)$ & $396(7)$ & $264(6)$ & $51(7)$ & $5(4)$ & $4(8)$ & $559(7)$ & $153(5)$ \\
\hline Pain & $484(4)$ & $304(5)$ & $149(3)$ & $28(4)$ & $3(3)$ & $2(4)$ & 349 (4) & $133(5)$ \\
\hline Fatigue/malaise & $395(4)$ & $248(4)$ & $115(3)$ & $29(4)$ & $3(3)$ & $3(6)$ & $260(3)$ & $132(5)$ \\
\hline Discomfort & $201(2)$ & $116(2)$ & $70(2)$ & $11(2)$ & $4(3)$ & $3(6)$ & $145(2)$ & $53(2)$ \\
\hline
\end{tabular}

$A A$ anaplastic astrocytoma, $A E$ adverse event, $A O$ oligodendroglioma, MedDRA Medical Dictionary for Regulatory Activities, $n d G B M$ newly diagnosed glioblastoma, $r G B M$ recurrent glioblastoma, TTFields Tumor Treating Fields

${ }^{a}$ Commonly described as a tingling sensation beneath the transducer arrays

${ }^{b}$ Local heat beneath the transducer array, commonly described as a warm sensation

${ }^{\mathrm{c}}$ Other brain tumors including brain metastases from different cancer types 
infrequent across all subgroups and can also be a result of or attributable to underlying brain cancer disease symptomology (4-8\%; Table 3).

Other device-related skin AEs included hyperhidrosis, wound complication (including dehiscence and infection), hypersensitivity, and skin erosions/ulcers (each $\leq 1 \%$ ) (Table 4). Moreover, only 27 unique patients $(<1 \%)$ experienced serious device-related skin AEs (Table 5). Serious skin AEs included wound complication, skin erosion, skin ulcer, and skin laceration. Rates of balance disorders, falls, and fractures that were deemed related to TTFields device usage were low and comparable for all diagnostic and age subgroups (each $\leq 1 \%$ ). To assess the potential impact of device-related falls on increased age-related incidences, a breakdown of number of falls by age distribution in decades (years of age; 11-20, 21-30, 31-40, 41-50, 51-60, 61-70, 71-80, and 81-90) was calculated. The percent ratios of patients with TTFields device-related falls to total falls by age distribution in decades were $0 \%, 11 \%, 0 \%, 6 \%, 9 \%, 9 \%$, $4 \%$, and $8 \%$, respectively.

Moreover, only $0-1 \%$ of patients across subgroups reported an AE of TTFields-related 'quality of life (QoL) decreased'. It is important to note that 'QoL decreased', in the context of these analyses, refers to an unsolicited AE incidence of 'QoL decreased' per MedDRA v21.1 preferred terms and is not by any means a reference to any standardized, validated QoL assessment.

\section{Discussion}

We retrospectively assessed the safety of TTFields in realworld, clinical practice settings, using global post-marketing surveillance data from a large patient cohort. Patient diagnoses included ndGBM, rGBM, and off-label treatment for anaplastic astrocytoma and anaplastic oligodendroglioma as well as metastatic brain tumors. Also, the male-to-female ratio of 1.97:1 was generally representative of global GBM populations, although slightly on the higher limit of the range. This slightly increased male predominance perhaps could be attributable to a number of factors, including sex hormones playing a relevant role, more women who do not want to shave their heads than men, or general population variability during the period that patients were retrospectively evaluated. It is important to note that the presumption that more women than men do not agree to shaving their head for aesthetic purposes, to noninvasively treat a very aggressive form of cancer, has not been evaluated to date and is speculative. Our results confirm the overall tolerability of TTFields, reveal no new safety signals, and demonstrate comparable safety and tolerability to that reported in the phase 3 TTFields/GBM clinical trials. As expected from our experiences with the EF-11 [21] and EF-14 [11] clinical trials and the PRiDe US post-marketing registry [28], mild-to-moderate localized skin AEs beneath the transducer arrays are the most commonly reported TTFieldsassociated AEs. Interestingly, there is no Common Terminology Criteria for AE (CTCAE) to date that is close to grading the severity of skin reactions, inclusive of irritant contact dermatitis. However, it is noteworthy to mention that a modified TTFields-specific skin reaction grading system is typically utilized to more accurately grade the severity of skin AEs, such as irritant contact dermatitis. Overall, it is comparable to the general AE severity grading guidelines. The modified grading system for TTFields-related skin reaction severity are divided into 4 categories. Grading categories are defined as: (1) Grade 1 (mild/asymptomatic symptoms)—no intervention required or only topical treatment intervention indicated; treatment interruption of $<3$ days may be required; (2) Grade 2 (moderate symptoms)—systemic therapy required or event is requiring interruption of TTFields for $>3$ days; (3) Grade 3 (severe/medically significant)—not immediately life threatening; hospitalization or prolongation of existing hospitalization indicated; and (4) Grade 4 (life threatening consequences)—urgent intervention indicated.

Skin AEs manifest as localized skin reactions beneath the transducer arrays. As previously reported in the literature, these skin AEs (irritant contact dermatitis) are most probably related to chronic skin exposure to irritants in transducer arrays, which are applied noninvasively to deliver TTFields through the skin to the tumor bed [29-31]. AE rates were comparable between adult and elderly patients and were lower in pediatric patients, likely due to the smaller pediatric sample size. The frequency of patients reporting $\geq 1 \mathrm{AE}$ were similar across ndGBM, rGBM, and other gliomas, suggesting no observable differences between pathologic diagnosis and AE rates. Similar to results from clinical trials, no notable TTFields-related systemic AEs were detected. For example, in the EF-14 ndGBM trial, TTFields plus TMZ combination did not lead to increased systemic AEs compared with TMZ alone, suggesting that systemic toxicity was TMZ-related [11].

These data suggest tolerability and the feasibility of longterm TTFields treatment exposure to potentially improve treatment adherence and patient outcomes. As the overall TTFields dose is also related to usage and has been shown to correlate directly with OS, these data are significant for demonstrating that treatment adherence is critical for improved survival outcomes [32,33]. In this context, it is worth noting that the effects of TTFields cease when the device system is powered off and that TTFields have no half-life, bioavailability, drug-drug interactions, or other pharmacokinetic parameters to consider.

In our analysis, $\mathrm{AE}$ incidence in general was lowest in pediatric patients (most likely associated with the smaller 
Table 4 TTFields-related AEs, by diagnosis and age (full data set)

\begin{tabular}{|c|c|c|c|c|c|c|c|c|}
\hline \multirow[t]{2}{*}{ MedDRA v21.1 system organ class/preferred term } & \multirow[t]{2}{*}{ Total } & \multicolumn{4}{|l|}{ Diagnosis } & \multicolumn{3}{|c|}{ Age (years) } \\
\hline & & ndGBM & $\mathrm{rGBM}$ & $\mathrm{AA} / \mathrm{AO}$ & Other ${ }^{\mathrm{c}}$ & $<18$ & 18 to 64 & $\geq 65$ \\
\hline $\mathbf{N} / \mathbf{n}$ & $\mathrm{N}=11,029$ & $\mathrm{n}=5887$ & $\mathrm{n}=4345$ & $\mathrm{n}=682$ & $\mathrm{n}=115$ & $\mathrm{n}=52$ & $\mathrm{n}=8090$ & $\mathrm{n}=2887$ \\
\hline Patients with $\geq 1$ AE, n (\%) & $5260(48)$ & $3022(51)$ & $1835(42)$ & $347(51)$ & $56(49)$ & $27(52)$ & $3842(47)$ & $1391(48)$ \\
\hline Ear, n (\%) & $3(<1)$ & $2(<1)$ & - & $1(<1)$ & - & - & $1(<1)$ & $2(<1)$ \\
\hline Auditory disorder & $3(<1)$ & $2(<1)$ & - & $1(<1)$ & - & - & $1(<1)$ & $2(<1)$ \\
\hline Eye, n (\%) & $3(<1)$ & $3(<1)$ & - & - & - & - & $2(<1)$ & $1(<1)$ \\
\hline Eye disorder & $3(<1)$ & $3(<1)$ & - & - & - & - & $2(<1)$ & $1(<1)$ \\
\hline Gastrointestinal, n (\%) & $6(<1)$ & $3(<1)$ & $2(<1)$ & - & $1(1)$ & - & $6(<1)$ & - \\
\hline Nausea/vomiting & $6(<1)$ & $3(<1)$ & $2(<1)$ & - & $1(1)$ & - & $6(<1)$ & - \\
\hline General, n (\%) & $2683(24)$ & $1533(26)$ & $936(22)$ & $183(27)$ & $31(27)$ & $16(31)$ & $2013(25)$ & $654(23)$ \\
\hline Chills & $1(<1)$ & - & - & $1(<1)$ & - & - & - & $1(<1)$ \\
\hline Complication associated with device & $4(<1)$ & $3(<1)$ & $1(<1)$ & - & - & - & $1(<1)$ & $3(<1)$ \\
\hline Discomfort & $201(2)$ & $116(2)$ & $70(2)$ & $11(2)$ & $4(3)$ & $3(6)$ & $145(2)$ & $53(2)$ \\
\hline Electric sensation $^{\mathrm{a}}$ & $1169(11)$ & $673(11)$ & $399(9)$ & $77(11)$ & $20(17)$ & $7(13)$ & $945(12)$ & $217(8)$ \\
\hline Fatigue/malaise & $395(4)$ & $248(4)$ & $115(3)$ & $29(4)$ & $3(3)$ & $3(6)$ & $260(3)$ & $132(5)$ \\
\hline Gait disturbance & $8(<1)$ & $4(<1)$ & $4(<1)$ & - & - & - & $7(<1)$ & $1(<1)$ \\
\hline Heat sensation $^{\mathrm{b}}$ & $1153(10)$ & $619(11)$ & $439(10)$ & $86(13)$ & $9(8)$ & $9(17)$ & $862(11)$ & $282(10)$ \\
\hline Edema & $4(<1)$ & $4(<1)$ & - & - & - & - & $2(<1)$ & $2(<1)$ \\
\hline Edema peripheral & $8(<1)$ & $8(<1)$ & - & - & - & - & $7(<1)$ & $1(<1)$ \\
\hline Pain & $484(4)$ & $304(5)$ & $149(3)$ & $28(4)$ & $3(3)$ & $2(4)$ & $349(4)$ & $133(5)$ \\
\hline Pyrexia & $1(<1)$ & - & $1(<1)$ & - & - & - & $1(<1)$ & - \\
\hline QoL decreased & $77(1)$ & $61(1)$ & $14(<1)$ & $2(<1)$ & - & - & $58(1)$ & $19(1)$ \\
\hline Immune system, n (\%) & $16(<1)$ & $15(<1)$ & - & $1(<1)$ & - & - & $13(<1)$ & $3(<1)$ \\
\hline Hypersensitivity & $16(<1)$ & $15(<1)$ & - & $1(<1)$ & - & - & $13(<1)$ & $3(<1)$ \\
\hline Infections, n (\%) & $11(<1)$ & $9(<1)$ & $1(<1)$ & $1(<1)$ & - & - & $8(<1)$ & $3(<1)$ \\
\hline Abscess & $2(<1)$ & $1(<1)$ & $1(<1)$ & - & - & - & $2(<1)$ & - \\
\hline Infection & $9(<1)$ & $8(<1)$ & - & $1(<1)$ & - & - & $6(<1)$ & $3(<1)$ \\
\hline Injury, n (\%) & $156(1)$ & $92(2)$ & $53(1)$ & $9(1)$ & $2(2)$ & - & $103(1)$ & $53(2)$ \\
\hline Contusion & $18(<1)$ & $13(<1)$ & $5(<1)$ & - & - & - & $14(<1)$ & $4(<1)$ \\
\hline Fall & $52(<1)$ & $22(<1)$ & $24(1)$ & $6(1)$ & - & - & $34(<1)$ & $18(1)$ \\
\hline Fracture & $3(<1)$ & $3(<1)$ & - & - & - & - & $3(<1)$ & - \\
\hline Injury & $9(<1)$ & $6(<1)$ & $3(<1)$ & - & - & - & $5(<1)$ & $4(<1)$ \\
\hline Muscle strain & $1(<1)$ & $1(<1)$ & - & - & - & - & $1(<1)$ & - \\
\hline Radiation injury & $1(<1)$ & $1(<1)$ & - & - & - & - & $1(<1)$ & - \\
\hline Skin abrasion & $2(<1)$ & $1(<1)$ & $1(<1)$ & - & - & - & - & $2(<1)$ \\
\hline Skin laceration & $42(<1)$ & $27(<1)$ & $13(<1)$ & $2(<1)$ & - & - & $27(<1)$ & $15(<1)$ \\
\hline Wound complication & $46(<1)$ & $29(<1)$ & $13(<1)$ & $2(<1)$ & $2(2)$ & - & $33(<1)$ & $13(<1)$ \\
\hline Musculoskeletal, n (\%) & $37(<1)$ & $15(<1)$ & $19(<1)$ & $3(<1)$ & - & - & $30(<1)$ & $7(<1)$ \\
\hline Muscle spasms & $5(<1)$ & $4(<1)$ & $1(<1)$ & - & - & - & $4(<1)$ & $1(<1)$ \\
\hline Muscle twitching & $24(<1)$ & $8(<1)$ & $13(<1)$ & $3(<1)$ & - & - & $21(<1)$ & $3(<1)$ \\
\hline Muscular weakness & $7(<1)$ & $3(<1)$ & $4(<1)$ & - & - & - & $4(<1)$ & $3(<1)$ \\
\hline Musculoskeletal stiffness & $1(<1)$ & - & $1(<1)$ & - & - & - & $1(<1)$ & - \\
\hline Nervous system, n (\%) & $739(7)$ & $406(7)$ & $275(6)$ & $52(8)$ & $6(5)$ & $4(8)$ & $580(7)$ & $155(5)$ \\
\hline Balance disorder & $7(<1)$ & $5(<1)$ & $2(<1)$ & - & - & - & $7(<1)$ & - \\
\hline Cognitive disorder & $2(<1)$ & $1(<1)$ & $1(<1)$ & - & - & - & $2(<1)$ & - \\
\hline Coordination abnormal & $1(<1)$ & - & $1(<1)$ & - & - & - & $1(<1)$ & - \\
\hline Dizziness & $3(<1)$ & $3(<1)$ & - & - & - & - & $3(<1)$ & - \\
\hline Dysesthesia & $14(<1)$ & $6(<1)$ & $6(<1)$ & $1(<1)$ & $1(1)$ & - & $12(<1)$ & $2(<1)$ \\
\hline Headache & $716(6)$ & $396(7)$ & $264(6)$ & $51(7)$ & $5(4)$ & $4(8)$ & $559(7)$ & $153(5)$ \\
\hline
\end{tabular}


Table 4 (continued)

\begin{tabular}{|c|c|c|c|c|c|c|c|c|}
\hline \multirow[t]{2}{*}{ MedDRA v21.1 system organ class/preferred term } & \multirow[t]{2}{*}{ Total } & \multicolumn{4}{|l|}{ Diagnosis } & \multicolumn{3}{|c|}{ Age (years) } \\
\hline & & ndGBM & rGBM & $\mathrm{AA} / \mathrm{AO}$ & Other ${ }^{\mathrm{c}}$ & $<18$ & 18 to 64 & $\geq 65$ \\
\hline Hyperesthesia & $1(<1)$ & - & - & $1(<1)$ & - & - & $1(<1)$ & - \\
\hline Paresthesia & $3(1)$ & $3(<1)$ & - & - & - & - & $3(<1)$ & - \\
\hline Syncope & $1(<1)$ & - & $1(<1)$ & - & - & - & $1(<1)$ & - \\
\hline Psychiatric, n (\%) & $76(1)$ & $53(1)$ & $20(<1)$ & $3(<1)$ & - & - & $54(1)$ & $22(1)$ \\
\hline Agitation & $7(<1)$ & $3(<1)$ & $4(<1)$ & - & - & - & $4(<1)$ & $3(<1)$ \\
\hline Anxiety & $7(<1)$ & $4(<1)$ & $3(<1)$ & - & - & - & $4(<1)$ & $3(<1)$ \\
\hline Claustrophobia & $3(<1)$ & $2(<1)$ & - & $1(<1)$ & - & - & $3(<1)$ & - \\
\hline Confusional state & $1(<1)$ & $1(<1)$ & - & - & - & - & $1(<1)$ & - \\
\hline Depression & $8(<1)$ & $6(<1)$ & $2(<1)$ & - & - & - & $7(<1)$ & $1(<1)$ \\
\hline Insomnia & $50(<1)$ & $39(1)$ & $9(<1)$ & $2(<1)$ & - & - & $35(<1)$ & $15(1)$ \\
\hline Mental status change & $1(<1)$ & - & $1(<1)$ & - & - & - & $1(<1)$ & - \\
\hline Mood altered & $1(<1)$ & $1(<1)$ & - & - & - & - & $1(<1)$ & - \\
\hline Sleep disorder & $1(<1)$ & - & $1(<1)$ & - & - & - & $1(<1)$ & - \\
\hline Stress & $3(<1)$ & $2(<1)$ & $1(<1)$ & - & - & - & $2(<1)$ & $1(<1)$ \\
\hline Skin, n (\%) & $3895(35)$ & $2300(39)$ & $1295(30)$ & $264(39)$ & $36(31)$ & $19(37)$ & $2817(35)$ & 1059 (37) \\
\hline Alopecia & $3(<1)$ & $3(<1)$ & - & - & - & - & $3(<1)$ & - \\
\hline Decubitus ulcer & $2(<1)$ & $1(<1)$ & - & $1(<1)$ & - & $1(2)$ & - & $1(<1)$ \\
\hline Hyperhidrosis & $144(1)$ & $90(2)$ & $45(1)$ & $9(1)$ & - & - & $115(1)$ & $29(1)$ \\
\hline Skin erosion & $4(<1)$ & $3(<1)$ & $1(<1)$ & - & - & - & $2(<1)$ & $2(<1)$ \\
\hline Skin reaction & 3805 (34) & $2251(38)$ & 1258 (29) & $260(38)$ & $36(31)$ & $19(37)$ & 2748 (34) & $1038(36)$ \\
\hline Skin ulcer & $80(1)$ & $41(1)$ & $32(1)$ & $6(1)$ & $1(1)$ & $1(2)$ & $50(1)$ & $29(1)$ \\
\hline Hypersensitivity & $16(<1)$ & $15(<1)$ & - & $1(<1)$ & - & - & $13(<1)$ & $3(<1)$ \\
\hline Vascular, n (\%) & $2(<1)$ & $1(<1)$ & $1(<1)$ & - & - & - & - & $2(<1)$ \\
\hline Hematoma & $1(<1)$ & $1(<1)$ & - & - & - & - & - & $1(<1)$ \\
\hline Hemorrhage & $1(<1)$ & - & $1(<1)$ & - & - & - & - & $1(<1)$ \\
\hline
\end{tabular}

$A A$ anaplastic astrocytoma, $A E$ adverse event, $A O$ anaplastic oligodendroglioma, MedDRA Medical Dictionary for Regulatory Activities, $n d G B M$ newly diagnosed glioblastoma, $Q o L$ quality-of-life, $r G B \mathrm{M}$ recurrent glioblastoma, TTFields Tumor Treating Fields

${ }^{a}$ Commonly described as a tingling sensation beneath the transducer arrays

${ }^{b}$ Local heat beneath the transducer array, commonly described as a warm sensation

${ }^{\mathrm{c}}$ Other brain tumors including brain metastases from different cancer types

pediatric sample size). Observed AE incidences were comparable in the larger sample sizes of adult and elderly patients. In particular, incidence of skin reactions were similar for pediatric, adult, and elderly patients. TTFieldsrelated $\mathrm{AE}$ rates of balance disorders, falls, and fractures across age groups were low ( $\leq 1 \%$ each; Table 4$)$. These data also suggest that TTFields usage did not lead to excess AEs among elderly patients. Furthermore, the percent ratios of patients with TTFields device-related falls to total falls suggests no observable age-related patterns or incremental increases in device-related falls in geriatric subgroups relative to other age subgroups. Moreover, elderly patients showed the lowest observed incidence of both electric and heat sensations, headache, and discomfort across age subgroups. Based on these observations, KPS and Eastern Cooperative Oncology Group Performance Score (ECOG-PS), as well as the Lansky score for children and the Global Assessment of Functioning scores or other patient-related outcomes/factors may be more relevant and better scaled indicators for TTFields usage by patients with brain cancers in comparison to age. These performance status scales may be better means to quantify each cancer patient's general well-being and daily-life activities to determine feasibility of TTFields treatment. TTFields treatment of elderly patient with ndGBM has been described as promising, since TTFields plus adjuvant chemotherapy compared with chemotherapy alone suggests improved OS in elderly patients with ndGBM. However, further evidence on QoL and tolerability in elderly patients is needed [34]. Our real-world evidence of evaluated safety/tolerability data for TTFields in elderly patients with GBM aids in defining unmet needs in this select population. Given these positive benefits and aforementioned increased disease incidence in the geriatric population, 
Table 5 Incidence of TTFields-related serious AEs in the total patient cohort

\begin{tabular}{ll}
\hline MedDRA v21.1 system organ class/preferred term & Total \\
& $\mathrm{N}=11,029$ \\
\hline Patients with $\geq \mathbf{1}$ reported AE, $\mathbf{n}(\%)$ & $34(<1)$ \\
Injury, $\mathbf{n}(\%)$ & $24(<1)$ \\
Contusion & $1(<1)$ \\
Fall & $2(<1)$ \\
Fracture & $2(<1)$ \\
Radiation injury & $1(<1)$ \\
Skin laceration & $2(<1)$ \\
Wound complication & $19(<1)$ \\
Skin, $\mathbf{n}(\%)$ & $7(<1)$ \\
Skin erosion & $2(<1)$ \\
Skin ulcer & $5(<1)$ \\
Nervous system, $\mathbf{n}(\%)$ & $3(<1)$ \\
Headache & $3(<1)$ \\
Infections, $\mathbf{n}(\%)$ & $1(<1)$ \\
Abscess & $1(<1)$ \\
\hline
\end{tabular}

$A E$ adverse event, MedDRA medical dictionary for regulatory activities, TTFields Tumor Treating Fields

TTFields show potential as a feasible and favorable treatment modality for elderly patients with GBM.

TTFields-associated AEs are very different from chemotherapy-related AEs, a critical consideration in a patient population burdened with disease and symptomology, such as debilitating neurological symptoms and deficits elicited by the tumor itself [35] and unpleasant and sometimes lifethreatening AEs related to radiation, chemotherapy, and other investigational treatments $[36,37]$. To date, TTFields as a locoregional treatment modality do not induce systemic toxicity. Chemotherapy-treated patients commonly suffer from hematologic, gastrointestinal, and renal toxicity, as well as severe nausea, vomiting, headache, and fatigue [38]. In contrast, an EF-14 secondary analysis found that concomitant TTFields with TMZ in patients with ndGBM did not result in reduced health-related QoL (HRQoL) versus TMZ alone, except for increased incidence of itchy skin, which was expected [39]. This lack of negative influence on HRQoL further supports the utility of combining TTFields with other GBM treatments [39].

This study has a large cohort as its major strength, despite limitations based on its retrospective design. Analyses were not statistically powered based on study design and therefore comparative statements should be deemed observational in nature. For instance, AEs were reported as serious or nonserious with no AE grading system, since data were not collected from a clinical trial. TTFields should be administered continuously; however patient adherence to treatment was not specified and perhaps may have impacted occurrence of related AEs. In addition, we did not report on concurrent and concomitant therapies that may have likely inflated AE incidences; such as steroid usage, which could affect the fragility of skin in relation to TTFields application. Since safety data were collated only from TTFields-treated patients that reported AEs, incidence of the overall cohort and subgroups is likely overestimated. Finally, no efficacy, survival, or standardized QoL assessment data were included.

\section{Conclusions}

This retrospective analysis of post-marketing surveillance data, obtained from real-world clinical practice settings in $>11,000$ patients, is the largest dataset to date of patients with brain cancer treated with TTFields. Moreover, it is one of the largest datasets of patients with GBM/ high-grade glioma. TTFields treatment shows a favorable safety profile with localized, mild-to-moderate skin reactions that are often resoluble with over-the-counter topical ointments or by temporarily withholding TTFields treatment. Furthermore, no systemic effects or other treatmentrelated AEs were reported. As expected, the AE profile was comparable to published phase 3 TTFields/GBM clinical trial data and prior registry observations. Overall, the safety profile of TTFields remained consistent among patient subgroups (region, diagnosis, and age) and the total cohort, suggesting feasibility in multiple subpopulations, including elderly patients. These real-world global safety data confirm the known safety and tolerability of TTFields for GBM treatment.

Author contributions All authors contributed to the development of this primary manuscript, wrote, edited, and reviewed the drafts, and approved the final manuscript. Writing and editorial support was provided by Huda Ismail Abdullah, PhD, Global Publications, Novocure Inc. Also, Katie Groschwitz, PhD, of Spark Medica Inc, provided writing support and editorial assistance, which was funded by Novocure Inc. Safety support was provided by Julia Stindl, $\mathrm{PhD}$ and Sharon Perez, PhD of the Novocure Ltd Medical Safety department. Biostatistical support was provided by Gitit Lavy-Shahaf, $\mathrm{PhD}$ of the Novocure Ltd Biostatistics department.

Funding Not applicable.

Data availability The datasets generated during and/or analyzed during the current study are available 3 years after date of publication.

Code availability Not applicable.

\section{Compliance with ethical standards}

Conflicts of interest Wenyin Shi reported grants/research support from Novocure Inc, Brainlab AG, and Regeneron Pharmaceuticals; and work as a consultant/independent contractor for Novocure Inc, Varian Medical Systems Inc, and Brainlab AG. Deborah T Blumenthal reported clinical research grants/research support from Merck Sharp 
\& Dohme Corp; role as an advisory/board member and work as a consultant/independent contractor with Vascular Biogenics Ltd (VBL) and ViruCure Therapeutics; and role on speaker bureaus and honorarium from Takeda and AstraZeneca. Nancy AO Bush had no disclosures to report. Sied Kebir reported work on a speaker bureau and honorarium from Novocure Inc. Rimas V Lukas reported grants/research support from NCI P50CA221747, BrainUp grant 2136, Bristol-Myers Squibb (drug support only for IIT) and Ziopharm Oncology; role as an advisory/board member for Monteris Medical and Novocure Inc; work as a consultant/independent contractor for AbbVie Inc and Eisai Co; work on a speaker bureau for Novocure Inc; honorarium from MedLink Neurology, EBSCO Publishing, American Physician Institute, Clinical Care Options, and AbbVie; and travel accommodations from Novocure Inc. Yoshihiro Muragaki reported grants/research support from Merck Sharp \& Dohme Corp, Daiichi-Sankyo, Chugai Pharmaceutical Co, Otsuka Pharmaceutical Inc, Eisai Co, and Hitachi Co; work as a consultant/independent contractor for AbbVie Inc, Novocure Inc, Ono Pharmaceuticals, and Daiichi-Sankyo; and work on speaker bureaus for Merck Sharp \& Dohme Corp, Daiichi-Sankyo, Chugai Pharmaceutical Co, Otsuka Pharmaceutical Inc, Eisai Co, Novartis, Hitachi Co, and Bristol-Myers Squibb. Jay-Jiguang Zhu reported grants/research support from NCI/NRG Oncology, Boston Biomedical of Sumitomo Dainippon Pharma Global Oncology, Five Prime Therapeutics Inc, Immuno-Cellular Therapeutics LTD, Novocure Inc, and Tocagen Inc; role as an advisory/board member for Tocagen Inc; and honorarium from Asian Society of Neuro-oncology (ASNO). Martin Glas reported work as a consultant/independent contractor with Roche Pharma, Novartis, AbbVie Inc, Novocure Inc, and Daiichi-Sankyo; honorarium from Novartis, UCB Inc, TEVA Pharmaceuticals, Bayer Corp, Novocure Inc, Medac, Merck Sharp \& Dohme Corp, and Kyowa Kirin Group; and travel fees from Novocure Inc and Medac.

Ethics approval Not applicable.

Consent to participate Not applicable.

Consent for publication Not applicable.

Open Access This article is licensed under a Creative Commons Attribution 4.0 International License, which permits use, sharing, adaptation, distribution and reproduction in any medium or format, as long as you give appropriate credit to the original author(s) and the source, provide a link to the Creative Commons licence, and indicate if changes were made. The images or other third party material in this article are included in the article's Creative Commons licence, unless indicated otherwise in a credit line to the material. If material is not included in the article's Creative Commons licence and your intended use is not permitted by statutory regulation or exceeds the permitted use, you will need to obtain permission directly from the copyright holder. To view a copy of this licence, visit http://creativecommons.org/licenses/by/4.0/.

\section{References}

1. Collaborators GBaOCC (2019) Global, regional, and national burden of brain and other CNS cancer, 1990-2016: a systematic analysis for the Global Burden of Disease Study 2016. Lancet Neurol 18:376-393. https://doi.org/10.1016/s1474-4422(18)30468-x

2. Ostrom QT, Cioffi G, Gittleman H, Patil N, Waite K, Kruchko C, Barnholtz-Sloan JS (2019) CBTRUS statistical report: primary brain and other central nervous system tumors diagnosed in the United States in 2012-2016. Neuro Oncol 21:v1-v100. https:// doi.org/10.1093/neuonc/noz150
3. Louis DN, Perry A, Reifenberger G, von Deimling A, FigarellaBranger D, Cavenee WK, Ohgaki H, Wiestler OD, Kleihues P, Ellison DW (2016) The 2016 world health organization classification of tumors of the central nervous system: a summary. Acta Neuropathol 131:803-820. https://doi.org/10.1007/s0040 1-016-1545-1

4. Hanif F, Muzaffar K, Perveen K, Malhi SM, Simjee SU (2017) Glioblastoma multiforme: a review of its epidemiology and pathogenesis through clinical presentation and treatment. Asian Pac J Cancer Prev 18:3-9. https://doi.org/10.22034/APJCP .2017.18.1.3

5. Tian M, Ma W, Chen Y, Yu Y, Zhu D, Shi J, Zhang Y (2018) Impact of gender on the survival of patients with glioblastoma. Biosci Rep. https://doi.org/10.1042/bsr20180752

6. Shergalis A, Bankhead A 3rd, Luesakul U, Muangsin N, Neamati $\mathrm{N}$ (2018) Current challenges and opportunities in treating glioblastoma. Pharmacol Rev 70:412-445. https://doi.org/10.1124/ pr.117.014944

7. Stupp R, Mason WP, van den Bent MJ, Weller M, Fisher B, Taphoorn MJ, Belanger K, Brandes AA, Marosi C, Bogdahn U, Curschmann J, Janzer RC, Ludwin SK, Gorlia T, Allgeier A, Lacombe D, Cairncross JG, Eisenhauer E, Mirimanoff RO (2005) Radiotherapy plus concomitant and adjuvant temozolomide for glioblastoma. N Engl J Med 352:987-996. https://doi. org/10.1056/NEJMoa043330

8. Lukas RV, Wainwright DA, Ladomersky E, Sachdev S, Sonabend AM, Stupp R (2019) Newly diagnosed glioblastoma: a review on clinical management. Oncology 33:91-100

9. Lukas RV, Mrugala MM (2017) Pivotal therapeutic trials for infiltrating gliomas and how they affect clinical practice. Neurooncol Pract 4:209-219. https://doi.org/10.1093/nop/npw016

10. The National Comprehensive Cancer Network ${ }^{\circledR}$ (2019) NCCN Clinical Practice Guidelines in Oncology (NCCN Guidelines $\left.{ }^{\circledR}\right)$ : Central Nervous System Cancers. Version 3.2019. https://www. nccn.org/professionals/physician_gls/pdf/cns.pdf. Accessed 18 Oct 2019

11. Stupp R, Taillibert S, Kanner A, Read W, Steinberg D, Lhermitte B, Toms S, Idbaih A, Ahluwalia MS, Fink K, Di Meco F, Lieberman F, Zhu JJ, Stragliotto G, Tran D, Brem S, Hottinger A, Kirson ED, Lavy-Shahaf G, Weinberg U, Kim CY, Paek SH, Nicholas G, Bruna J, Hirte H, Weller M, Palti Y, Hegi ME, Ram Z (2017) Effect of tumor-treating fields plus maintenance temozolomide vs maintenance temozolomide alone on survival in patients with glioblastoma: a randomized clinical trial. JAMA 318:2306-2316. https://doi.org/10.1001/jama.2017.18718

12. Kirson ED, Dbalý V, Tovaryš F, Vymazal J, Soustiel JF, Itzhaki A, Mordechovich D, Steinberg-Shapira S, Gurvich Z, Schneiderman R, Wasserman Y, Salzberg M, Ryffel B, Goldsher D, Dekel E, Palti Y (2007) Alternating electric fields arrest cell proliferation in animal tumor models and human brain tumors. Proc Natl Acad Sci USA 104:10152-10157. https://doi.org/10.1073/pnas.07029 16104

13. Giladi M, Schneiderman RS, Voloshin T, Porat Y, Munster M, Blat R, Sherbo S, Bomzon Z, Urman N, Itzhaki A, Cahal S, Shteingauz A, Chaudhry A, Kirson ED, Weinberg U, Palti Y (2015) Mitotic spindle disruption by alternating electric fields leads to improper chromosome segregation and mitotic catastrophe in cancer cells. Sci Rep 5:18046. https://doi.org/10.1038/ srep18046

14. Kirson ED, Gurvich Z, Schneiderman R, Dekel E, Itzhaki A, Wasserman Y, Schatzberger R, Palti Y (2004) Disruption of cancer cell replication by alternating electric fields. Can Res 64:3288-3295

15. Giladi M, Schneiderman RS, Porat Y, Munster M, Itzhaki A, Mordechovich D, Cahal S, Kirson ED, Weinberg U, Palti Y (2014) Mitotic disruption and reduced clonogenicity of pancreatic cancer 
cells in vitro and in vivo by tumor treating fields. Pancreatology 14:54-63. https://doi.org/10.1016/j.pan.2013.11.009

16. Dizon DS, Krilov L, Cohen E, Gangadhar T, Ganz PA, Hensing TA, Hunger S, Krishnamurthi SS, Lassman AB, Markham MJ, Mayer E, Neuss M, Pal SK, Richardson LC, Schilsky R, Schwartz GK, Spriggs DR, Villalona-Calero MA, Villani G, Masters G (2016) Clinical Cancer Advances 2016: Annual Report on Progress Against Cancer From the American Society of Clinical Oncology. J Clin Oncol 34:987-1011. https://doi.org/10.1200/ jco.2015.65.8427

17. Kreisl TN, Kim L, Moore K, Duic P, Royce C, Stroud I, Garren N, Mackey M, Butman JA, Camphausen K, Park J, Albert PS, Fine HA (2009) Phase II trial of single-agent bevacizumab followed by bevacizumab plus irinotecan at tumor progression in recurrent glioblastoma. J Clin Oncol 27:740-745. https://doi.org/10.1200/ jco.2008.16.3055

18. Vredenburgh JJ, Desjardins A, Herndon JE 2nd, Marcello J, Reardon DA, Quinn JA, Rich JN, Sathornsumetee S, Gururangan S, Sampson J, Wagner M, Bailey L, Bigner DD, Friedman AH, Friedman HS (2007) Bevacizumab plus irinotecan in recurrent glioblastoma multiforme. J Clin Oncol 25:4722-4729. https://doi. org/10.1200/jco.2007.12.2440

19. Batchelor TT, Mulholland P, Neyns B, Nabors LB, Campone M, Wick A, Mason W, Mikkelsen T, Phuphanich S, Ashby LS, Degroot J, Gattamaneni R, Cher L, Rosenthal M, Payer F, Jurgensmeier JM, Jain RK, Sorensen AG, Xu J, Liu Q, van den Bent M (2013) Phase III randomized trial comparing the efficacy of cediranib as monotherapy, and in combination with lomustine, versus lomustine alone in patients with recurrent glioblastoma. J Clin Oncol 31:3212-3218. https://doi.org/10.1200/ jco.2012.47.2464

20. Wick W, Puduvalli VK, Chamberlain MC, van den Bent MJ, Carpentier AF, Cher LM, Mason W, Weller M, Hong S, Musib L, Liepa AM, Thornton DE, Fine HA (2010) Phase III study of enzastaurin compared with lomustine in the treatment of recurrent intracranial glioblastoma. J Clin Oncol 28:1168-1174. https://doi. org/10.1200/jco.2009.23.2595

21. Stupp R, Wong ET, Kanner AA, Steinberg D, Engelhard H, Heidecke V, Kirson ED, Taillibert S, Liebermann F, Dbalý V, Ram Z, Villano JL, Rainov N, Weinberg U, Schiff D, Kunschner L, Raizer J, Honnorat J, Sloan A, Malkin M, Landolfi JC, Payer F, Mehdorn M, Weil RJ, Pannullo SC, Westphal M, Smrcka M, Chin L, Kostron H, Hofer S, Bruce J, Cosgrove R, Paleologous N, Palti Y, Gutin PH (2012) NovoTTF-100A versus physician's choice chemotherapy in recurrent glioblastoma: a randomised phase III trial of a novel treatment modality. Eur J Cancer 48:2192-2202. https://doi.org/10.1016/j.ejca.2012.04.011

22. Taal W, Oosterkamp HM, Walenkamp AM, Dubbink HJ, Beerepoot LV, Hanse MC, Buter J, Honkoop AH, Boerman D, de Vos FY, Dinjens WN, Enting RH, Taphoorn MJ, van den Berkmortel FW, Jansen RL, Brandsma D, Bromberg JE, van Heuvel I, Vernhout RM, van der Holt B, van den Bent MJ (2014) Single-agent bevacizumab or lomustine versus a combination of bevacizumab plus lomustine in patients with recurrent glioblastoma (BELOB trial): a randomised controlled phase 2 trial. Lancet Oncol 15:943-953. https://doi.org/10.1016/s1470-2045(14)70314-6

23. van Linde ME, Brahm CG, de Witt Hamer PC, Reijneveld JC, Bruynzeel AME, Vandertop WP, van de Ven PM, Wagemakers M, van der Weide HL, Enting RH, Walenkamp AME, Verheul HMW (2017) Treatment outcome of patients with recurrent glioblastoma multiforme: a retrospective multicenter analysis. J Neurooncol 135:183-192. https://doi.org/10.1007/s11060-017-2564-Z

24. Kesari S, Ram Z (2017) Tumor-treating fields plus chemotherapy versus chemotherapy alone for glioblastoma at first recurrence: a post hoc analysis of the EF-14 trial. CNS Oncol 6:185-193. https ://doi.org/10.2217/cns-2016-0049
25. Stupp R, Dietrich P-Y, Kraljevic SO, Pica A, Maillard I, Maeder P, Meuli R, Janzer R, Pizzolato G, Miralbell R, Porchet F, Regli L, Tribolet Nd, Mirimanoff RO, Leyvraz S (2002) Promising Survival for Patients With Newly Diagnosed Glioblastoma Multiforme Treated With Concomitant Radiation Plus Temozolomide Followed by Adjuvant Temozolomide. J Clin Oncol 20:1375-1382. https://doi.org/10.1200/jco.2002.20.5.1375

26. Perry JR, Belanger K, Mason WP, Fulton D, Kavan P, Easaw J, Shields C, Kirby S, Macdonald DR, Eisenstat DD, Thiessen B, Forsyth P, Pouliot JF (2010) Phase II trial of continuous doseintense temozolomide in recurrent malignant glioma: RESCUE study. J Clin Oncol 28:2051-2057. https://doi.org/10.1200/ JCO.2009.26.5520

27. Gilbert MR, Wang M, Aldape KD, Stupp R, Hegi ME, Jaeckle KA, Armstrong TS, Wefel JS, Won M, Blumenthal DT, Mahajan A, Schultz CJ, Erridge S, Baumert B, Hopkins KI, Tzuk-Shina T, Brown PD, Chakravarti A, Curran WJ Jr, Mehta MP (2013) Dose-dense temozolomide for newly diagnosed glioblastoma: a randomized phase III clinical trial. J Clin Oncol 31:4085-4091. https://doi.org/10.1200/jco.2013.49.6968

28. Mrugala MM, Engelhard HH, Dinh Tran D, Kew Y, Cavaliere R, Villano JL, Annenelie Bota D, Rudnick J, Love Sumrall A, Zhu J-J, Butowski N (2014) Clinical practice experience with NovoTTF-100A TM System for glioblastoma: the patient registry dataset (PRiDe). Semin Oncol 41:S4-S13. https://doi. org/10.1053/j.seminoncol.2014.09.010

29. Lacouture M, Iwamoto F, Armentano F, Kim E (2016) Mitigating skin irritations with tumor treating fields therapy in glioblastoma. Oncology nursing society annual conference, Washington, DC

30. Lacouture ME, Davis ME, Elzinga G, Butowski N, Tran D, Villano JL, DiMeglio L, Davies AM, Wong ET (2014) Characterization and management of dermatologic adverse events with the NovoTTF-100A System, a novel anti-mitotic electric field device for the treatment of recurrent glioblastoma. Semin Oncol 41(suppl 4):S1-14. https://doi.org/10.1053/j.seminoncol.2014.03.011

31. Lukas RV, Ratermann KL, Wong ET, Villano JL (2017) Skin toxicities associated with tumor treating fields: case based review. J Neurooncol 135:593-599. https://doi.org/10.1007/s1106 $0-017-2612-8$

32. Ballo MT, Urman N, Lavy-Shahaf G, Grewal J, Bomzon Z, Toms S (2019) Correlation of tumor treating fields dosimetry to survival outcomes in newly diagnosed glioblastoma: a large-scale numerical simulation-based analysis of data from the phase 3 EF-14 randomized trial. Int J Radiat Oncol Biol Phys 104:1106-1113. https ://doi.org/10.1016/j.ijrobp.2019.04.008

33. Toms SA, Kim CY, Nicholas G, Ram Z (2019) Increased compliance with tumor treating fields therapy is prognostic for improved survival in the treatment of glioblastoma: a subgroup analysis of the EF-14 phase III trial. J Neurooncol 141:467-473. https://doi. org/10.1007/s11060-018-03057-z

34. Hanna C, Lawrie TA, Rogozinska E, Kernohan A, Jefferies S, Bulbeck H, Ali UM, Robinson T, Grant R (2020) Treatment of newly diagnosed glioblastoma in the elderly: a network metaanalysis. Cochrane Database Syst Rev 3:CD013261. https://doi. org/10.1002/14651858.CD013261.pub2

35. Omuro A, DeAngelis LM (2013) Glioblastoma and other malignant gliomas: a clinical review. JAMA 310:1842-1850. https:// doi.org/10.1001/jama.2013.280319

36. Villano JL, Letarte N, Yu JM, Abdur S, Bressler LR (2012) Hematologic adverse events associated with temozolomide. Cancer Chemother Pharmacol 69:107-113. https://doi.org/10.1007/s0028 0-011-1679-8

37. Simonetti G, Trevisan E, Silvani A, Gaviani P, Botturi A, Lamperti E, Beecher D, Bertero L, Bosa C, Salmaggi A (2014) Safety of bevacizumab in patients with malignant gliomas: a systematic 
review. Neurol Sci 35:83-89. https://doi.org/10.1007/s1007 2-013-1583-6

38. Bae SH, Park MJ, Lee MM, Kim TM, Lee SH, Cho SY, Kim YH, Kim YJ, Park CK, Kim CY (2014) Toxicity profile of temozolomide in the treatment of 300 malignant glioma patients in Korea. J Korean Med Sci 29:980-984. https://doi.org/10.3346/ jkms.2014.29.7.980

39. Taphoorn MJB, Dirven L, Kanner AA, Lavy-Shahaf G, Weinberg U, Taillibert S, Toms SA, Honnorat J, Chen TC, Sroubek J, David C, Idbaih A, Easaw JC, Kim CY, Bruna J, Hottinger AF, Kew Y, Roth P, Desai R, Villano JL, Kirson ED, Ram Z, Stupp R (2018)
Influence of treatment with tumor-treating fields on health-related quality of life of patients with newly diagnosed glioblastoma: a secondary analysis of a randomized clinical trial. JAMA Oncol 4:495-504. https://doi.org/10.1001/jamaoncol.2017.5082

Publisher's Note Springer Nature remains neutral with regard to jurisdictional claims in published maps and institutional affiliations.

\section{Affiliations}

\section{Wenyin Shi ${ }^{1}$ (I) - Deborah T. Blumenthal ${ }^{2} \cdot$ Nancy Ann Oberheim Bush ${ }^{3} \cdot$ Sied Kebir $^{4,5} \cdot$ Rimas V. Lukas $^{6,7}$. Yoshihiro Muragaki ${ }^{8,9} \cdot$ Jay-Jiguang Zhu ${ }^{10} \cdot$ Martin Glas ${ }^{4,5}$}

1 Department of Radiation Oncology, Thomas Jefferson University, Philadelphia, PA 19107, USA

2 Neuro-Oncology Unit, Division of Oncology, Tel Aviv Sourasky Medical Center and Sackler School of Medicine, Tel Aviv University, Tel Aviv, Israel

3 Department of Neurological Surgery and Neurology, University of California San Francisco, San Francisco, CA, USA

4 Division of Clinical Neurooncology, Department of Neurology, University Hospital Essen, University Duisburg-Essen, Essen, Germany

5 West German Cancer Center, German Cancer Consortium, Partner Site Essen, University Hospital Essen, University Duisburg-Essen, Essen, Germany
6 Department of Neurology, Northwestern University, Chicago, IL, USA

7 Lou \& Jean Malnati Brain Tumor Institute at the Lurie Comprehensive Cancer Center, Northwestern University, Chicago, USA

8 Faculty of Advanced Techno-Surgery, Institute of Advanced Biomedical Engineering and Science, Tokyo Women's Medical University, Tokyo, Japan

9 Department of Neurosurgery, Tokyo Women's Medical University, Tokyo, Japan

10 McGovern Medical School and Memorial Hermann Hospital at Texas Medical Center, University of Texas Health Science Center at Houston, Houston, TX, USA 